\title{
Matrix Gla protein limits pulmonary arteriovenous malformations in ALK1 deficiency
}

\begin{abstract}
To the Editor:
Hereditary haemorrhagic telangiectasia (HHT), also referred to as the Osler-Weber-Rendu syndrome, is characterised by multiple arteriovenous malformations (AVMs) in different organs including lungs, brain, liver, intestine and spleen [1]. Five types of HHT have been identified [1]. Mutations in the gene for the activin receptor-like kinase 1 (Alk1), a bone morphogenetic protein (BMP) type I receptor, are known to cause HHT2 [2]. Loss of function studies in mice have demonstrated that deletion of Alk1 results in embryonic lethality due to AVMs in early embryogenesis [3]. However, heterozygous $A l k 1^{+/-}$mice are viable and develop AVMs at a similar rate as humans with HHT2 [3-5].

The expression of ALK1 is regulated by BMP4 and matrix Gla protein (MGP), a BMP inhibitor, via feedback regulation [6-8]. Excess MGP inhibits the growth of the pulmonary vasculature through its inhibitory effect on BMP4 [9], whereas loss of MGP increases BMP activity and causes pulmonary AVMs in $M g p^{-l-}$ mice, despite increased pulmonary ALK1 expression [8]. Since deficiency of ALK1 is associated with decreased MGP expression [8], we hypothesised that enhancing MGP rather than ALK1 would limit AVMs in ALK1-deficient mice.
\end{abstract}

To determine the effect of increased MGP on pulmonary AVMs in $A l k 1^{+/-}$mice, we crossbred $A l k 1^{+/-}$ mice with MGP transgenic $\left(M g p^{t g / w t}\right)$ mice to obtain $A l k 1^{+/-} M g p^{t g / w t}$ mice. The investigation conformed to the National Research Council Guidelines for the Care and Use of Laboratory Animals [10], and was approved by the Institutional Review Board of the University of California, Los Angeles. All the mice had a C57BL/6 background. We grouped the mice as wild type control $(\mathrm{n}=28), M g p^{t g / w t}(\mathrm{n}=28), A l 1^{+/-}$ $(\mathrm{n}=27)$ and $A l k 1^{+/-} M g p^{t g / w t}(\mathrm{n}=31)$ mice. The mice were maintained for 1 year to allow for AVM formation [4]. The lungs were then screened for AVMs by injection of fluorescent microspheres followed by UV detection $[8,11]$. The test was considered to be positive for AVMs if the intensity was $<5 \%$ of that the wild type mouse with the strongest fluorescence, as assessed using Image J software (US National Institutes of Health, Bethesda, MD, USA; http://imagej.nih.gov/ij/). The intensity in all other mice ranged between 50 and $100 \%$ of the wild type control. We discovered that four out of $27 \mathrm{Alk} 1^{+/-}$mice $(14.8 \%)$ harboured pulmonary AVMs (fig. 1a). This is higher than previously reported [4] potentially due to differences in the background of the mice or the method of AVM detection. No pulmonary AVMs were detected in the $A l k^{+/-} M g p^{t g / w t}$, the $M g p^{t g / w t}$, or the wild type mice suggesting that the excess MGP prevented AVMs in the $A l k 1^{+-}$mice. Lungs from each of the mouse groups are shown in Figure 1b; lungs with AVMs are shown for the $A l k 1^{+/-}$mice.

We then examined the pulmonary vasculatures of five additional mice of each type by micro-computed tomography (CT) imaging (images from three-dimensional reconstructions are shown in fig. 1c). Several signs indicated pulmonary $\mathrm{AVMs}$ in one of the $A l k 1^{+/-}$mice, including abnormal arteriovenous shunts, often referred to as niduses, and visualisation of the venous system due to shunting of contrast. In $A l k 1^{+/-} M g p^{t g / w t}$ mice, we neither detected niduses nor visualised the venous system. Analysis of the vessel radii in the micro-CT reconstructions showed an increase in the frequency of all vessel radii between 10 and $100 \mu \mathrm{m}$ in the $A l k 1^{+/-}$mouse with AVMs (fig. 1d). Interestingly, the $A l k 1^{+/-}$mice without AVMs did not differ significantly from wild type mice, suggesting that factor(s) other than ALK1 deficiency are necessary to trigger AVMs.

Less vascular branching was seen in $A l k 1^{+/-} M g p^{t g / w t}$ mice compared with wild type controls (fig. 1c) suggesting that the $A l k 1^{+/-} M g p^{t g / w t}$ mice develop a phenotype similar to that of $M g p^{t g / w t}$ mice [8]. Furthermore, the frequency of vessel radii smaller than $60 \mu \mathrm{m}$ was significantly decreased in the lungs of both $M g p^{t g / w t}$ and $A l k 1^{+/-} M g p^{t g / w t}$ mice, as compared with wild type controls (fig. 1d).

Because earlier studies suggested low MGP levels in $A l k 1^{+/-}$mice [12], we examined the expression of MGP in the lungs, which had previously been examined by microsphere injection, of wild type, $M g p^{t g / w t}$, $A l k 1^{+/-}$and $A l k 1^{+/-} M g p^{t g / w t}$ mice by real-time PCR. We used 4-5 sets of lungs in each group. For Alk $1^{+/-}$ mice, the four sets with AVMs were processed separately from the five sets without AVMs. We found that the pulmonary MGP expression in $A l k 1^{+/-}$mice was significantly lower than in wild type regardless of whether AVMs were absent or present (fig. le shows results from the lungs with AVMs for the $A l k 1^{+/-}$mice). 

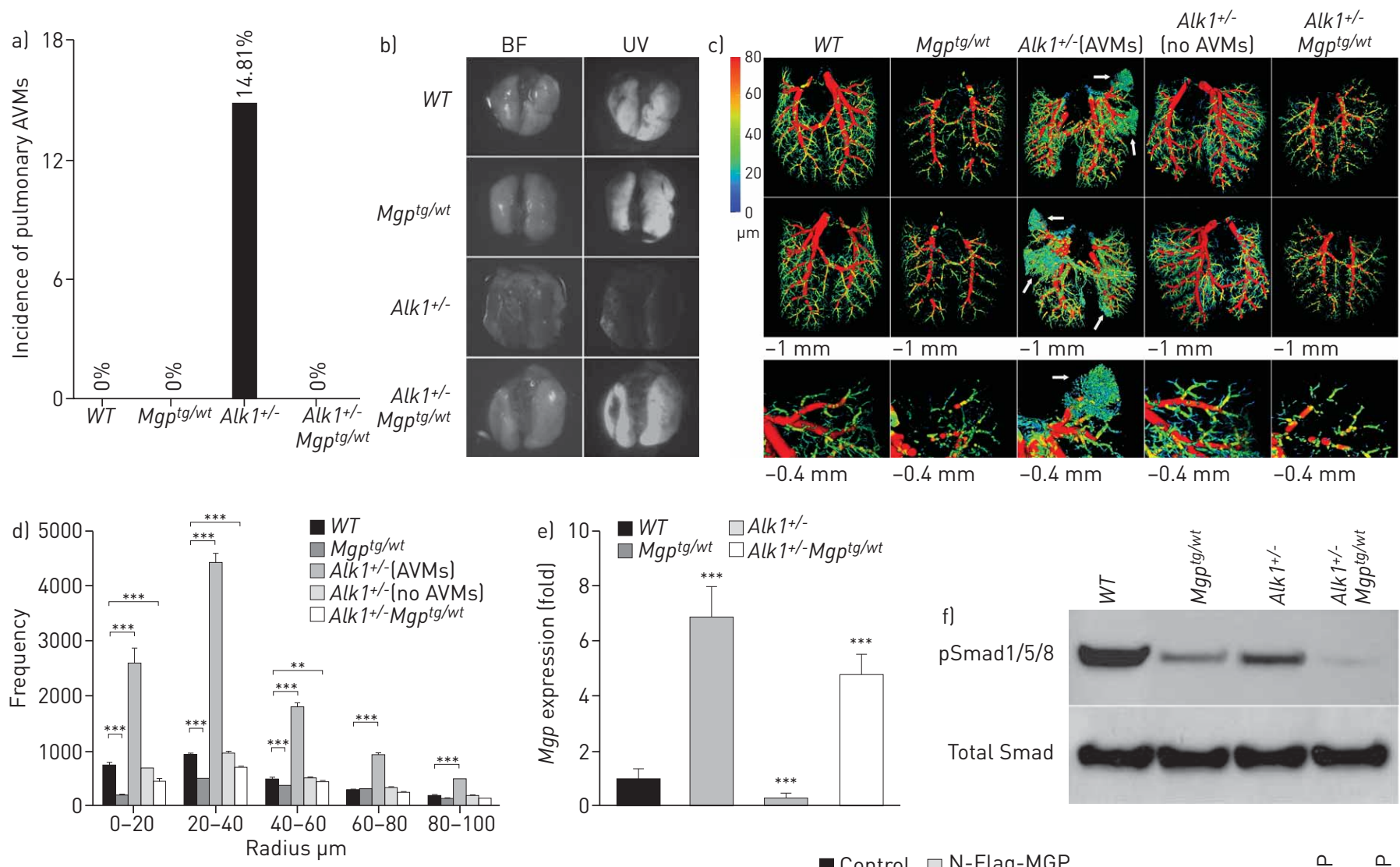

f)

$\mathrm{pSmad} 1 / 5 / 8$

Total Smad

- WT $\square A l k 1^{+1-}$

$\square M g p^{t g / w t} \square A l k 1+/-M g p^{t g} / w t$
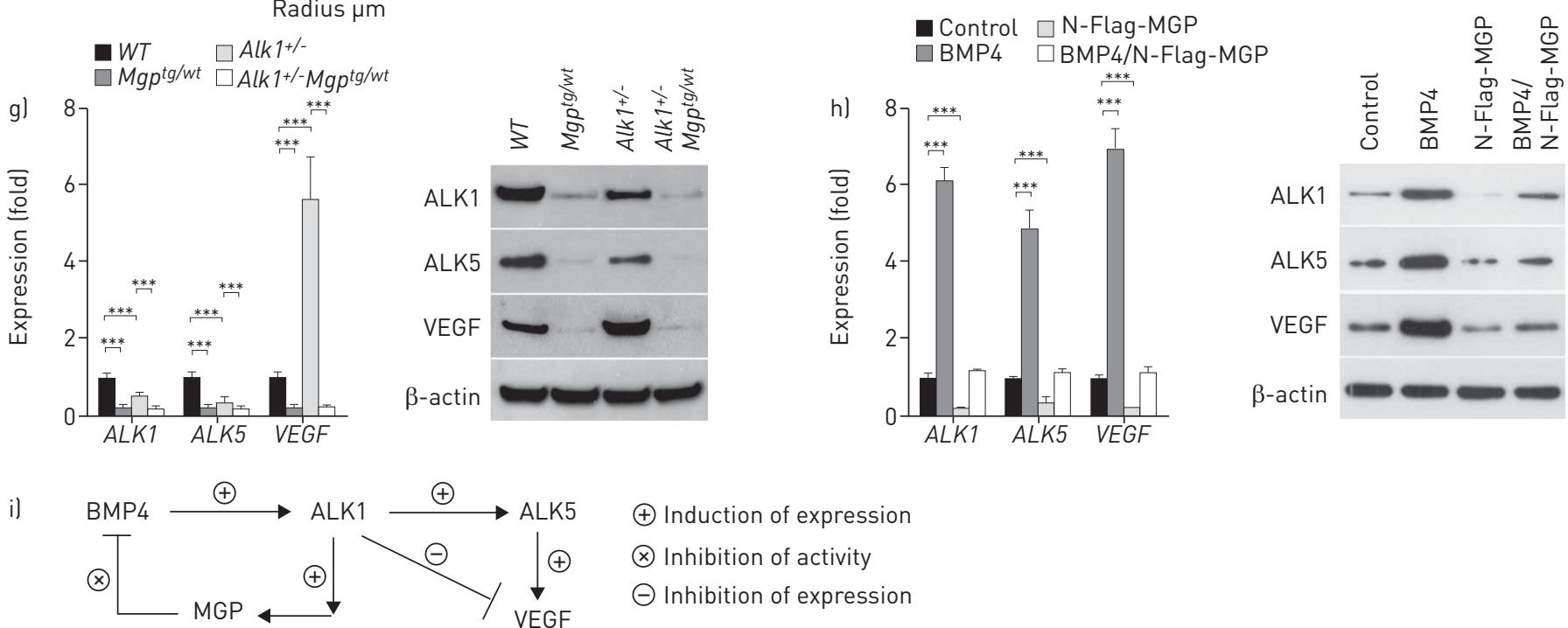

$\oplus$ Induction of expression

$\otimes$ Inhibition of activity

$\Theta$ Inhibition of expression

FIGURE 1 Increased matrix Gla protein (MGP) levels decrease arteriovenous malformations (AVMs) in the lungs of $A l k 1^{+/-} M g p^{t g / w t}$ mice. a) Incidence of pulmonary AVMs in wild type (WT) control mice $(\mathrm{n}=28), M g p^{\text {tg/wt }}$ mice $(\mathrm{n}=28), A l k 1^{+/-}$mice $(\mathrm{n}=27)$, and $A l k 1^{+/-} M g p^{\text {tg/wt }}$ mice $(\mathrm{n}=31)$. b) Example of pulmonary arteriovenous shunting by UV-fluorescent microsphere passage in one of the $A l k 1^{+/-}$mice with AVMs, shown together with lungs from WT, Mgg ${ }^{\text {tg/wt }}$ and $A l k 1^{+/-} M g p^{t g / w t}$ mice (left panels: bright field (BF); right panels: UV). c) Micro-computed tomography images of the pulmonary vasculature from WT, $M g p^{t g / w t}$, $A l k 1^{+-}$with AVMs, $A l k 1^{+-}$without AVMs and $A l k 1^{+-} M g p^{t g / w t}$ mice. Arrows indicate visualised niduses of pulmonary AVMs. See Supplemental Movies 1-4 for full three-dimensional reconstructions of the pulmonary vasculature. d) Radius analysis of the pulmonary vasculatures of the WT, $M g p^{\text {tg/wt }}, A l k 1^{+/-}$with AVMs, $A l k 1^{+/-}$without AVMs and $A l k 1^{+/-} M g p^{\text {tg/wt }}$ mice shown in panel c. Bars represent the mean of four independent determinations in each radius range. e) Expression of $M g p$ in the lungs of WT, $M g p^{t g / w t}, A l k 1^{+1-}$, and $A l k 1^{+-} M g p^{t g / w t}$ mice, as determined by real-time PCR. f) Phosphorylated (p)SMAD1/5/8 in lung tissues from WT, $M g p^{t g / w t}, A l k 1^{+/-}$, and $A l k 1^{+/-} M g p^{t g / w t}$ mice, as determined by immunoblotting and compared with total SMAD. g) Expression of activin

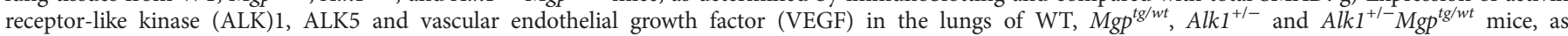
determined by real-time PCR and immunoblotting. $\beta$-actin was used as a loading control. h) Mouse pulmonary vascular endothelial cells were treated with bone morphogenetic protein (BMP)4 $\left(40 \mathrm{ng} \cdot \mathrm{mL}^{-1}\right)$, transfected with N-Flag-tagged MGP, or both treated and transfected. After $24 \mathrm{~h}$, expression of ALK1, ALK5 and VEGF was assessed by real-time PCR (left) and immunoblotting (right). $\beta$-actin was used as a loading control. i) Working model of the relationships between BMP4, ALK1, ALK5, MGP and VEGF. Data were analysed for statistical significance by ANOVA with post hoc Tukey's analysis. The analyses were performed using GraphPad Instat, version 3.0 (GraphPad Software, Inc., La Jolla, CA, USA). ${ }^{* *}$ : p $<0.01 ;{ }^{* * *}$ : $<<0.001$. 
However, MGP expression in both $A l k 1^{+/-} M g p^{t g / w t}$ and $M g p^{t g / w t}$ mice was 5-7-fold higher than in wild type mice (fig. 1e); supporting the hypothesis that high MGP expression may limit the development of AVMs.

SMAD1/5/8 is phosphorylated in response to BMPs signalling. In previous studies phosphorylated $(\mathrm{p})$ SMAD1/5/8 decreased in the lungs of $M g p^{t g / w t}$ mice due to reduced BMP4 activity [9]. To assess BMP activity, we examined the pSMAD1/5/8 levels in the lungs of the four mouse groups by immunoblotting. This revealed a significant decrease in pSMAD1/5/8 in the $M g p^{t g / w t}$ and $A l k 1^{+/-} M g p^{t g / w t}$ mice, compared with wild type and $A l k 1^{+/-}$mice (fig. 1f). The pSMAD1/5/8 level was also lower in $A l k 1^{+/-}$mice compared with wild type (fig. 1f), probably due to the reduced ALK1 levels. There was no difference between $A l k 1^{+/-}$mice with and without AVMs.

We then examined the pulmonary expression of ALK1, ALK5 and vascular endothelial growth factor (VEGF) in the different mice by real-time PCR and immunoblotting. Previous studies showed that MGP interferes with BMP4 stimulation of endothelial cell proliferation, which is mediated by VEGF $[8,9]$. Furthermore, ALK5 is induced by ALK1 signalling, and in turn enhances VEGF expression when stimulated by transforming growth factor- $\beta 1[8,12]$. However, it is also known that ALK1 directly suppresses VEGF expression by an unknown mechanism [12]. Therefore, patients with HHT have decreased ALK5, but enhanced VEGF levels [13]. In our studies, the expression of ALK1 and ALK5 declined in the $M g p^{t g / w t}, A l k 1^{+/-}$and $A l k 1^{+/-} M g p^{t g / w t}$ mice to $<50 \%$ of wild type controls (fig. $1 \mathrm{~g}$ ). There was no significant difference between the $M g p^{t g / w t}$ and the $A l k 1^{+/-} M g p^{t g / w t}$ mice (fig. $1 \mathrm{~g}$ ). As expected, the expression of VEGF was increased in the $A l k 1^{+/-}$mice (fig. 1g) consistent with previous data [12, 13], probably due to less suppression from ALK1. By contrast, VEGF expression was similarly reduced in both the $M g p^{t g / w t}$ and the $A l k 1^{+/-} M g p^{t g / w t}$ mice [12], suggesting that the increase in MGP overcame the lack of suppression from ALK1 in the $A l k 1^{+/-} M g p^{t g / w t}$ mice. Expression of ALK1 in the Alk1 ${ }^{+/-}$mice was about $50 \%$ of that in wild type mice as previously reported (fig. 1g) [3, 4], without significant differences between the $A l k 1^{+/-}$mice with and without AVMs.

To further demonstrate how BMP4 and MGP co-modulate expression of ALK1, ALK5 and VEGF, we treated mouse pulmonary vascular endothelial cells with BMP4, transfected them with N-Flag-tagged MGP [14], or combined the BMP4 treatment and the transfection. After $24 \mathrm{~h}$, we examined the expression of ALK1, ALK5 and VEGF by real-time PCR and immunoblotting. As expected, all three proteins were highly induced by BMP4 and suppressed by N-Flag-MGP (fig. 1h).

Altogether, our results suggest that excess MGP, as provided by an Mgp transgene, limits the formation of pulmonary AVMs in $A l k 1^{+/-}$mice. The $A l k 1^{+/-}$mice resemble the $M g p^{-/-}$mice in that both have reduced MGP and high VEGF. However, the increase in VEGF is caused by different mechanisms. In the $\mathrm{Mgp}^{-/-}$ mice, uninhibited BMP4 enhances expression of VEGF (fig. 1i) [8]. In $A l k 1^{+/-}$mice, the reduced ALK1 results in less suppression of VEGF expression [12].

Although VEGF is believed to contribute to AVMs in the lungs, this may not be sufficient to trigger AVMs. We propose that aberrant BMP activity is a necessary contributing factor. $M g p^{-1-}$ mice, with extensive vascular BMP activation, all have pulmonary AVMs [8]. The $A l k 1^{+/-}$mice have decreased levels of MGP, which would be consistent with a decreased induction of ALK1 and its target gene MGP in response to BMP activity [7]. Such a diminished response may be sufficient to regulate BMP under quiet conditions, but not enough to suppress BMP activity induced by, for example, disturbed flow and inflammation [15]. In such areas, AVMs could be triggered. This might explain the lower incidence of AVMs in the $A l k 1^{+/-}$mice and the apparent need for a "secondary insult" [16] to trigger AVMs.

0 @ERSpublications

Increased MGP limits pulmonary AVMs in ALK1 deficiency and may be modulated for the purpose of treating AVMs http://ow.ly/FadMt

Kristina I. Boström ${ }^{1,2}$, Pierre Guihard ${ }^{1}$, Ana M. Blazquez Medela ${ }^{1}$, Jiayi Yao ${ }^{1}$, Jeremiah H. Moon ${ }^{1}$, Ashley Penton ${ }^{1}$ and Yucheng Yao ${ }^{1}$

${ }^{1}$ Division of Cardiology, David Geffen School of Medicine at UCLA, Los Angeles, CA, USA. ${ }^{2}$ The Molecular Biology Institute at UCLA, Los Angeles, CA, USA.

Correspondence: Yucheng Yao, Division of Cardiology, David Geffen School of Medicine at UCLA, Box 951679, Los Angeles, CA 90095-1679, USA. E-mail: yyao@mednet.ucla.edu

Received: June 232014 | Accepted after revision: Nov 222014 | First published online: Jan 222014

This article has supplementary material available from erj.ersjournals.com

Support statement: Funding for this work was provided in part by US National Institutes of Health grants NS79353, HL30568, HL81397, and HL112839, and the American Heart Association (grant number: 10SDG3050043). Funding information for this article has been deposited with FundRef. 
Conflict of interest: None declared.

\section{References}

1 Abdalla SA, Letarte M. Hereditary haemorrhagic telangiectasia: current views on genetics and mechanisms of disease. J Med Genet 2006; 43: 97-110.

2 Johnson DW, Berg JN, Baldwin MA, et al. Mutations in the activin receptor-like kinase 1 gene in hereditary haemorrhagic telangiectasia type 2. Nat Genet 1996; 13: 189-195.

3 Urness LD, Sorensen LK, Li DY. Arteriovenous malformations in mice lacking activin receptor-like kinase-1. Nat Genet 2000; 26: 328-331.

4 Srinivasan S, Hanes MA, Dickens T, et al. A mouse model for hereditary hemorrhagic telangiectasia (HHT) type 2. Hum Mol Genet 2003; 12: 473-482.

5 Park SO, Wankhede M, Lee YJ, et al. Real-time imaging of de novo arteriovenous malformation in a mouse model of hereditary hemorrhagic telangiectasia. J Clin Invest 2009; 119: 3487-3496.

6 Yao Y, Jumabay M, Ly A, et al. Crossveinless 2 regulates bone morphogenetic protein 9 in human and mouse vascular endothelium. Blood 2012; 119: 5037-5047.

7 Yao Y, Zebboudj AF, Shao E, et al. Regulation of bone morphogenetic protein-4 by matrix GLA protein in vascular endothelial cells involves activin-like kinase receptor 1. J Biol Chem 2006; 281: 33921-33930.

8 Yao Y, Jumabay M, Wang A, et al. Matrix Gla protein deficiency causes arteriovenous malformations in mice. J Clin Invest 2011; 121: 2993-3004.

9 Yao Y, Nowak S, Yochelis A, et al. Matrix GLA protein, an inhibitory morphogen in pulmonary vascular development. J Biol Chem 2007; 282: 30131-30142.

10 National Research Council. Guide for the Care and Use of Laboratory Animals. 8th Edn. Washington, The National Academies Press, 2011.

11 Yao Y, Yao J, Radparvar M, et al. Reducing Jagged 1 and 2 levels prevents cerebral arteriovenous malformations in matrix Gla protein deficiency. Proc Natl Acad Sci USA 2013; 110: 19071-19076.

12 Shao ES, Lin L, Yao Y, et al. Expression of vascular endothelial growth factor is coordinately regulated by the activin-like kinase receptors 1 and 5 in endothelial cells. Blood 2009; 114: 2197-2206.

13 Fernandez LA, Garrido-Martin EM, Sanz-Rodriguez F, et al. Therapeutic action of tranexamic acid in hereditary haemorrhagic telangiectasia (HHT): regulation of ALK-1/endoglin pathway in endothelial cells. Thromb Haemost 2007; 97: 254-262.

14 Yao Y, Shahbazian A, Boström KI. Proline and gamma-carboxylated glutamate residues in matrix Gla protein are critical for binding of bone morphogenetic protein-4. Circ Res 2008; 102: 1065-1074.

15 Csiszar A, Lehoux S, Ungvari Z. Hemodynamic forces, vascular oxidative stress, and regulation of BMP-2/4 expression. Antioxid Redox Signal 2009; 11: 1683-1697.

16 Garrido-Martin EM, Nguyen HL, Cunningham TA, et al. Common and distinctive pathogenetic features of arteriovenous malformations in hereditary hemorrhagic telangiectasia 1 and hereditary hemorrhagic telangiectasia 2 animal models - brief report. Arterioscler Thromb Vasc Biol 2014; 34: 2232-2236. 